\title{
Neurological complications associated with the treatment of patients with congenital cardiac disease: consensus definitions from the Multi-Societal Database Committee for Pediatric and Congenital Heart Disease
}

\author{
Geoffrey L. Bird ${ }^{1}$, Howard E. Jeffries ${ }^{2}$, Daniel J. Licht ${ }^{3}$, Gil Wernovsky ${ }^{1}$, Paul M. \\ Weinberg ${ }^{4}$, Christian Pizarro ${ }^{5}$, and Giovanni Stellin 6 \\ ${ }^{1}$ Divisions of Pediatric Cardiology and Critical Care Medicine, The Children's Hospital of \\ Philadelphia and University of Pennsylvania School of Medicine, Philadelphia, Pennsylvania, United \\ States of America \\ ${ }^{2}$ Children's Hospital and Regional Medical Center, University of Washington School of Medicine, \\ Seattle, Washington, United States of America \\ ${ }^{3}$ Division of Neurology, The Children's Hospital of Philadelphia and University of Pennsylvania \\ School of Medicine, Philadelphia, Pennsylvania, United States of America \\ ${ }^{4}$ Division of Pediatric Cardiology, The Children's Hospital of Philadelphia and University of \\ Pennsylvania School of Medicine, Philadelphia, Pennsylvania, United States of America \\ ${ }^{5}$ Nemours Cardiac Center, Alfred I. duPont Hospital for Children, Jefferson Medical College, \\ Wilmington, Delaware, United States of America \\ ${ }^{6}$ Pediatric Cardiac Surgery Unit, University of Padova Medical School, Padova, Italy
}

\begin{abstract}
A complication is an event or occurrence that is associated with a disease or a healthcare intervention, is a departure from the desired course of events, and may cause, or be associated with suboptimal outcome. A complication does not necessarily represent a breech in the standard of care that constitutes medical negligence or medical malpractice. An operative or procedural complication is any complication, regardless of cause, occurring (1) within 30 days after surgery or intervention in or out of the hospital, or (2) after 30 days during the same hospitalization subsequent to the operation or intervention. Operative and procedural complications include both intraoperative/intraprocedural complications and postoperative/postprocedural complications in this time interval.
\end{abstract}

The MultiSocietal Database Committee for Pediatric and Congenital Heart Disease has set forth a comprehensive list of complications associated with the treatment of patients with congenital cardiac disease, related to cardiac, pulmonary, renal, haematological, infectious, neurological, gastrointestinal, and endocrine systems, as well as those related to the management of anaesthesia and perfusion, and the transplantation of thoracic organs. The objective of this manuscript is to examine the definitions of operative morbidity as they relate specifically to the neurological system. These specific definitions and terms will be used to track morbidity associated with surgical and

\footnotetext{
(C) Cambridge University Press

Correspondence to: Geoffrey L. Bird, MD MSIS FAAP, CICU Physician Office Suite, 6th Floor, Main Building, The Children's Hospital of Philadelphia, 324 South 34th Street, Philadelphia, Pennsylvania 19104-4399, United States of America. Tel: 215-590-6256; Fax: 215-590-1924; E-mail: bird@email.chop.edu.
} 
transcatheter interventions and other forms of therapy in a common language across many separate databases.

Although neurological injury and adverse neurodevelopmental outcome can follow procedures for congenital cardiac defects, much of the variability in neurological outcome is now recognized to be more related to patient specific factors rather than procedural factors. Additionally, the recognition of pre and postoperative neurological morbidity requires procedures and imaging modalities that can be resource-intensive to acquire and analyze, and little is known or described about variations in "sampling rate" from centre to centre.

The purpose of this effort is to propose an initial set of consensus definitions for neurological complications following congenital cardiac surgery and intervention. Given the dramatic advances in understanding achieved to date, and those yet to occur, this effort is explicitly recognized as only the initial first step of a process that must remain iterative. This list is a component of a systemsbased compendium of complications that may help standardize terminology and possibly enhance the study and quantification of morbidity in patients with congenital cardiac malformations. Clinicians caring for patients with congenital cardiac disease may be able to use this list for databases, initiatives to improve quality, reporting of complications, and comparing strategies of treatment.

\section{Keywords}

Congenital heart disease; quality improvement; patient safety; outcomes; registry; operative morbidity; paediatric; surgery; congenital abnormalities; cardiac surgical procedures; postoperative stroke

\section{Historical background}

As operative mortality after surgery for complex congenital cardiac disease continues to decrease, focus has shifted to efforts to recognize and mitigate other morbidities. Adverse neurodevelopmental outcome, and other neurological injuries, remain common after intervention for congenital cardiac disease. ${ }^{1}$ Early research efforts to decrease neurological injury focused on modifying intraoperative techniques of management, ${ }^{2}$ and advances in these techniques have been modestly successful. However, in the current era, surgeons and their teams are no longer solely responsible for further understanding and further advances. Many centres now recognize that non-modifiable patient-related factors are more important determinants of adverse neurodevelopmental outcome. ${ }^{3}$ With studies describing abnormal fetal brain development ${ }^{4}$ and brain perfusion, ${ }^{5}$ congenital cardiac disease is, in many cases, accompanied by congenital brain disease. 6,7

It seems reasonable that further advances in improving care will be aided by having a common language across disciplines for describing abnormal findings in the central nervous system recognized before, during, and after interventions for congenital cardiac disease. However, the creation of this common language to describe neurological complications associated with the treatment of patients with congenital cardiac disease is a very challenging area in which to tread. Even the frequently mentioned construct, "postoperative complication", gives the impression that the operation and/or the surgical team makes or breaks the occurrence of injury. For neurological disease, this assumption is simply not always true. Evidence is accumulating that multiple factors may be as important, or more important, than intraoperative factors in overall outcomes, including the factors from the following domains:

- $\quad$ Factors in the fetus

- Factors in the delivery room

- $\quad$ Preoperative factors in the intensive care unit 
- Postoperative factors in the intensive care unit.

Identifying perioperative neurological injury may be challenging. Normal findings on physical examination and diagnostic studies are poorly sensitive for longer term neurological dysfunction, and these normal findings can have limited predictive validity. Similarly, the specificity of early postoperative findings for long term neurological and developmental abnormalities is also limited, with surprising ability of the neonatal and infant brain to withstand and recover from perioperative injury. Most structural neurological injury in the perioperative period is diagnosed with imaging modalities of magnetic resonance imaging or computed tomography, while functional abnormalities are typically assessed by electroencephalography and physical examination. However, beyond routine physical examination, advanced assessment of structure and function are rarely carried out. The vast majority of seizures and strokes are clinically silent after cardiac surgery. The neonatal physical examination is widely regarded to be of little prognostic value in predicting neurological outcome. Given the limited sensitivity and specificity of these studies, clinicians must balance the risks and benefits of cost, time, utilization of human resources, and other potential risks in moving a critically ill patient for a lengthy neuroimaging study requiring complete immobilization for adequate imaging.

It is with this understanding that candidate definitions for neurological complications are presented as only the first step of the work required to improve care in this area. Standardizing every aspect of neurological care is beyond the scope of this work. As technology, care, and understanding advance, it is anticipated that these definitions will be in need of revision. We believe that a common language and definitions are likely to improve neurological care and outcomes of children with congenital cardiac disease. However, it is important to recognize that standardization may also have unintended consequences ${ }^{8}$ that are not yet foreseen.

\section{Consensus definitions}

The terms in the final list of neurological complications developed by The MultiSocietal Database Committee for Pediatric and Congenital Heart Disease, along with their official definitions, are listed in Part 4 of this Supplement.

\section{Controversies}

While many of the definitions were relatively straightforward to draft, some areas required further inquiry and discussion.

1. Many definitions do not allow for clarity or explanation that might be afforded by inclusion of aetiologies. As an example, coma and stupor are intended to be used to describe inherent pathology, and not a secondary effect from, for example, sedative drug administration. Including an exhaustive list of aetiologies for the various neurological complications would likely make the list inordinately long, complex, and difficult to use and difficult to validate. Without aetiologies, however, some question remains as to how best to code the neurological effect of certain medications. Possibilities include the following options:

- Adverse drug reaction

- Neurological complication — not otherwise specified

- Or, perhaps, the particular effect itself.

Also, omitting aetiologies may contribute to the risk, throughout the database, of different centres using different codes or terminology to describe the same fundamental complication. 
2. To some, the construct of "no deficit persisting at hospital discharge" is troubling from a clinical perspective. The reason it remains included is to enhance harmonization efforts with work from other societies and collaborations, for example, the Society for Thoracic Surgeons. Additionally, the authors recognize that many clinical and administrative databases can tend to view the concept of "the state of health at discharge from the hospital" as a critical parameter. It must be said, however, that from the point of view of the clinician, certain neurological deficits rarely, if ever, resolve prior to discharge. Furthermore, in infants, the neurological injury may be clinically silent at diagnosis, with symptoms that become apparent remotely, years after the acute hospitalization. Additionally, the resolution of symptoms may depend solely upon how hard the healthcare team tries to identify these residual effects.

Discharge itself, as a time frame, remains somewhat arbitrary and nebulous as a basis of comparison. Exceptional resources for the follow-up of the outpatient may make a given centre, clinician, or family, feel differently about a given discharge time-frame than would those at a less well equipped centre. This conundrum may lead to the same neurological complication being coded differently for reasons beyond the complication itself.

3. While "the pediatric patient population" may be construed by some as a single group of patients, the wide variation in neuroanatomy and neurophysiology between extremes of age within that group can hamper efforts to draft definitions of complications with universal applicability. Debate concerning the validity of neonatal physical examination in predicting neurodevelopmental outcome is well recognized. 9,10 Wherever feasible, neurological imaging studies should be used in extending the predictive validity of the neonatal neurological examination. The neurological evaluation of the preterm and full-term newborn infant represents a unique set of challenges as compared to the evaluation of older children and adults with congenital cardiac disease. As mentioned earlier, the dictionary of complications provided in Part 4 of this Supplement is meant to attempt to apply for patients of all ages with congenital cardiac disease, including adults with congenital cardiac disease.

4. The definitions include a non-specific term, "Neurological complication." The reason this term remains included is to act as a final choice for those rare complications which are not suitably described by one of the other terms in the list. Concern has been expressed about the possibility that this term could be used as a generic catch-all term, like "not otherwise specified." Auditing effort should occur to assure that this term does not get overused by participating centres with less than a full commitment to choosing the most detailed complication descriptors, or by those who might try to obscure the incidence of adverse complications with generic descriptors.

5. Blindness, deafness, and perhaps some other sensorineural complication descriptors appear in the list without the detail that might be expected by a domain expert. Understandably, a board-certified paediatric ophthalmologist might find the generality posed by the term "blindness" to be somewhat troublesome. Also, similar to definitions for renal failure and pulmonary disease in the work of other committees responsible for other organ systems, a mandate does not exist for formal subspecialty consultation to determine given criteria. Furthermore, the needs of efficiency make it beyond the scope of this work to have encyclopaedic representation of all subspecialties. If the presumed rates of postoperative blindness increase, or if there are other factors which mandate that additional detail or clarity be added to a given definition, these terms and related issues could fall within the purview of future work groups on future iterations of the set of definitions of complications.

6. The concept of developmental delay has been included in the set of definitions, but some committee members find its future utility suspect. It can be a difficult and time- 
consuming concept to quantitate with a particular metric, and almost any child with a hospital course of any length can be expected to meet the definition's criteria. Others on the committee found it to be of potential use in describing a pre-existing condition. It is hoped that auditing work in the future will serve to clarify the term's utility and to help determine whether revision, clarity, or elimination are warranted in future iterations of this set of definitions of complications.

7. Two factors have mandated the inclusion of certain terms with little or no relevance for paediatric medicine. The first is an imperative to maintain harmonization with databases from other societies. The second factor is that, as discussed above, the list of complications in Part 4 of this Supplement is meant to apply for patients of all ages with congenital cardiac disease, including adults with congenital cardiac disease. The following terms are some of those which may have more relevance for care of adults than children:

- Coma

- Delirium

- Reversible Ischemic Neurological Deficit (RIND).

Experts in paediatric neurology consulted for this work specifically portrayed the relevance of the term "Reversible Ischemic Neurological Deficit" as "waning" in the adult community and absent in the paediatric setting. This collaborative effort to discretely define complications must be reconciled with the fact that, as medical knowledge advances, the term "Reversible Ischemic Neurological Deficit", and perhaps other concepts, may get disused, outdated, or overtly replaced. The term is included in the interest of possibly meeting the needs of some centres caring for late adolescents and adults with congenital cardiac disease, but auditing work may help to clarify the necessity of this and similar terms remaining in future iterations - of both the paediatric and adult domains.

8. In Part 4 of this Supplement, the following definitions are provided for stroke, reversible ischemic neurologic deficit, and transient ischemic attack:

"A stroke is any confirmed neurological deficit of abrupt onset caused by a disturbance in blood flow to the brain, when the neurologic deficit does not resolve within 24 hours."

"A reversible ischemic neurologic deficit (RIND) is defined as the loss of neurological function with symptoms at least 24 hours after onset but with complete return of function within 72 hours. In other words, a transient disturbance of perfusion to a localized part of the brain which produces a temporary, focal lesion with defined deficiency of neurologic function lasting from 24 to 72 hours, but not resulting in sustained symptoms or injury. Because a stroke is defined as "any confirmed neurological deficit of abrupt onset caused by a disturbance in blood flow to the brain, when the neurologic deficit does not resolve within 24 hours", a RIND is a subtype of a stroke where the loss of neurological function and symptoms completely resolve within 72 hours."

"A transient ischemic attack (TIA) is the temporary loss of neurological function resulting from temporary occlusion of blood flow in a cerebral artery, but without resulting in permanent brain injury. Most symptoms last less than 5 minutes but may last hours - up to 24 hours."

In the past, the term "transient ischemic attack" was used to describe neurological deficits of less than 24 hours, the term "reversible ischemic neurologic deficit" was 
used to describe neurological deficits of 24 hours to 72 hours, and the term stroke was used to describe neurological deficits of greater than 72 hours. Now, the term "transient ischemic attack" is still used to describe neurological deficits of less than 24 hours; however, the term stroke is used to describe neurological deficits of greater than 24 hours. Therefore, those centres that choose to use the term "reversible ischemic neurologic deficit" are describing a rare type of stroke associated with a neurological deficit lasting between 24 hours to 72 hours.

In the current era, some feel that it is important to differentiate between duration of symptoms and changes on neurological imaging. Using this approach, the term Transient Ischemic Attack would be used to describe a stroke-like event whose symptoms resolve within 24 hours and which does not result in persistent abnormalities on neurological imaging. A stroke-like event which does result in persistent abnormalities on neurological imaging would be called a stroke, regardless of when the clinical symptoms resolve.

9. An effort has been made to include seizure in the set of definitions, but some have expressed concerns that lack of certain details may limit the utility of the term and contribute to errors in coding. One of the main issues lies with the extreme difficulty in defining discrete physical criteria for a "clinical" seizure. Even without discrete clinical criteria, the clinical assessment of seizure in infants is well described to be limited, ${ }^{11}$ at best, for this patient population. That being said, the term is included with clarifiers for electroencephalographic and clinical assessment, and with the individual ability to recognize or determine a clinical seizure left to the participating practitioner, team, or cardiac centre. The following terms are therefore defined in Part 4 of this Supplement:

- $\quad$ Seizure

- Seizure, Clinically silent with electroencephalography (EEG) confirmation

- $\quad$ Seizure, Clinically suspected with electroencephalography (EEG) confirmation

- $\quad$ Seizure, Clinically suspected without electroencephalography (EEG) confirmation:

- $\quad$ Seizure, New onset postoperatively/postprocedurally

- Seizure, Present preoperatively/preprocedurally and postoperatively/ postprocedurally.

10. Individual parts of the set of definitions may not strictly mimic the particular thought or work processes of a given centre. One such example is the manner in which the concept of "stroke" is included. In terms of the definitions, stroke is 1) first determined to exist, or not, then 2) localized to a particular area of the brain, and lastly 3) attributed to an underlying cause usually indicated by the particular imaging modality, for example, from thrombosis or haemorrhage. Variations in practice, at a given centre, and variations with approach, as medical knowledge is advanced, will require adaptability for both the user, and for the collaborators, on future iterations of this set of definitions of complications.

11. Despite the committee's best efforts, and those of the participating users, inevitable overlap in definitions will exist. In a centre without ready access to imaging, a postoperative stroke may be labelled as a coma, perhaps a seizure, or simply a "neurological complication." For the goals of completeness, cleanliness, and accuracy of the database, ideally, very explicit criteria will exist for each complication, with strictly clarified methods of ascertainment. However, there are other goals to achieve 
in this effort; these include ease of use, clarity, inclusion, workability, and the desire of this committee to make a first step in the right direction for the right reason. A certain degree of inaccuracy and "noise" in the system will occur secondary to multiple factors:

- Differences between institutions in the thresholds for a diagnosis

- Diagnosis by personnel other than subspecialists

- Variability in the access to and use of imaging modalities and electroencephalography, and

- Variability in access to neurological subspecialists.

It is hoped, but by no means guaranteed, that the gains of having this initial carefully thought out, but admittedly imperfect system, will outweigh the risks. Follow-up work, auditing, reassessment, and future iterative development will all be necessary.

\section{Interaction with the cardiac system}

Since the advent of paediatric cardiothoracic surgery, there has been an interesting evolution in the understanding of interactions between the neurological and cardiovascular systems of those affected by congenital cardiac disease. Early authors and sources ${ }^{12}$ lament the devastating effect on the developing brain of undiagnosed and untreatable cardiovascular disease. With profound circulatory collapse at presentation, or with the secondary effects of polycythemia and chronic cyanosis at later stages, the secondary effects on the brain were severe. With developments in surgical and medical therapies, efforts appropriately turned towards finding ways of minimizing the effects of cardiopulmonary bypass and other therapies. The current era involves improved rates of survival with efforts to mitigate the surprisingly high rate of adverse neurodevelopmental outcomes in the survivors. Careful work has shown that these adverse outcomes are multifactorial. In contrast to the model based solely in the operating room, it is now recognized that variation in intraoperative management fails to account for the majority of variability in neurodevelopmental outcomes. ${ }^{3}$ Through work on growth and development of the fetal brain, as well as preoperative and postoperative management in the intensive care unit, it is now clear that congenital cardiac disease rarely occurs in isolation many affected patients have pre-existing and lifelong secondary effects on their neurological systems. Postoperative neurological complications are, in the current era, probably only one small part of this overall spectrum of effect.

\section{Conclusion}

This manuscript represents a multidisciplinary, collaborative, initial effort to improve the global dialogue, reporting, and understanding of neurological complications associated with the treatment of patients with congenital cardiac disease. Despite unintended consequences that may occur with the limitations and controversies described above, it is hoped that this effort helps to achieve its true primary goal - a salutary effect on communication and collaboration towards better neurodevelopmental outcomes.

\section{Acknowledgement}

We thank The Children's Heart Foundation (http://www.childrensheartfoundation.org/) for financial support of this research. 


\section{References}

1. Ballweg JA, Wernovsky G, Gaynor JW. Neurodevelopmental outcomes following congenital heart surgery. Pediatr Cardiol 2007;28:126-133. [PubMed: 17265108]

2. Mahle WT, Wernovsky G. Neurodevelopmental outcomes after complex infant heart surgery. ACC Current Journal Review 2000;9:93-97.

3. Gaynor JW, Wernovsky G, Jarvik GP, et al. Patient characteristics are important determinants of neurodevelopmental outcome at one year of age after neonatal and infant cardiac surgery. $\mathrm{J}$ Thorac Cardiovasc Surg 2007;133:1344-1353. [PubMed: 17467455]

4. Licht DJ, Wang J, Silvestre DW, et al. Preoperative cerebral blood flow is diminished in neonates with severe congenital heart defects. J Thorac Cardiovasc Surg 2004;128:841-849. [PubMed: 15573068]

5. Kaltman JR, Di H, Tian Z, Rychik J. Impact of congenital heart disease on cerebrovascular blood flow dynamics in the fetus. Ultrasound Obstet Gynecol 2005;25:32-36. [PubMed: 15593334]

6. Wernovsky G. Improving neurologic and quality-of-life outcomes in children with congenital heart disease: past, present, and future. J Thorac Cardiovasc Surg 2008;135:240-242. [PubMed: 18242241]

7. Dominguez TE, Wernovsky G, Gaynor JW. Cause and prevention of central nervous system injury in neonates undergoing cardiac surgery. Semin Thorac Cardiovasc Surg 2007;19:269-277. [PubMed: 17983956]

8. Wachter RM. Expected and unanticipated consequences of the quality and information technology revolutions. JAMA 2006;295:2780-2783. [PubMed: 16788133]

9. Volpe JJ. Value of the neonatal neurologic examination. Pediatrics 1979;64:547-548. [PubMed: 492824]

10. Knight P. Value of the neonatal neurologic examination? Pediatrics 1980;66:150-151. [PubMed: 7402782]

11. Gaynor JW, Nicolson SC, Jarvik GP, et al. Increasing duration of deep hypothermic circulatory arrest is associated with an increased incidence of postoperative electroencephalographic seizures. J Thorac Cardiovasc Surg 2005;130:1278-1286. [PubMed: 16256779]

12. du Plessis AJ. Neurologic complications of cardiac disease in the newborn. Clin Perinatol 1997;24:807-826. [PubMed: 9395864] 